\title{
Blind Spots in Business and Information Systems Engineering
}

\author{
Oliver Hinz • Wil M. P. van der Aalst • \\ Christof Weinhardt
}

Published online: 20 February 2019

(C) Springer Fachmedien Wiesbaden GmbH, ein Teil von Springer Nature 2019

With a new editorial team and Oliver as new Vice-Editorin-Chief, the BISE Journal enters into a new phase of its development. The outgoing Editor-in-Chief, Martin Bichler, has done an excellent job in developing BISE into a more global journal with a growing reputation and standing as a premium journal for our field. As Martin leaves us with a thriving journal, we would like to use this opportunity and thank him and the outgoing Vice-Editors-in-Chief Armin Heinzl and all of their supporters for this outstanding work!

As new editors of our flagship journal for the BISE community, we are looking for blind spots that require more attention from our community. Such blind spots relate to issues that cannot be enlightened within a short period, as it will take us as a community some time to build up solid competences in those particular areas.

In the wake of all the changes enabled by the digital transformation as well as artificial intelligence and robotics, there is also a steadily increasing demand for exploring and quantifying the effects of such technologies on society

Prof. Dr. O. Hinz ( $\square)$

Faculty of Economics and Business Administration, Goethe University Frankfurt, Theodor-W.-Adorno-Platz 4,

60323 Frankfurt am Main, Germany

e-mail: ohinz@wiwi.uni-frankfurt.de

Prof. dr. ir. W. M. P. van der Aalst

Lehrstuhl für Informatik 9, RWTH Aachen, Ahornstr. 55,

52056 Aachen, Germany

e-mail: wvdaalst@pads.rwth-aachen.de

Prof. Dr. C. Weinhardt

Institute of Information Systems and Marketing (IISM),

Karlsruhe Institute of Technology (KIT), Fritz-Erler-Straße 23,

76133 Karlsruhe, Germany

e-mail: weinhardt@kit.edu and the economy as a whole. Thus, the demand for the competences of our community has never been greater.

It has always been a strength of our community to create innovative solutions to critical questions from business practice. Now, as large amounts of data are increasingly available to researchers, we also see an increasing number of scholars trying to understand the impact of (new) technology on different outcome variables based on empirical data analyses. This way, our community can find solutions to concrete operative problems while continuously getting better at developing theories that are generated around existent or emergent technologies.

At the same time, more and more people try to understand the implications of current developments in IT. Whenever we observe the rise of a new technology, business practitioners, politicians, the public and the media try to understand the impact on business and society. They turn to us and ask whether we can shed light on these questions. As scientists, we do not always feel comfortable to answer such questions. We usually look backward when it comes to understanding the impact of technology. One could argue that clairvoyance should not be the responsibility of a scientist from the Business and Information Systems Engineering community, but in this case, this argument is likely to fall short.

Think of our colleagues from the economics discipline. They usually deal with questions that can have substantial implications for policy-making. In doing so, economists usually rely on models that aim to capture the most important mechanisms behind a new phenomenon. These models can be false and sometimes misleading, but in most cases, they easily beat the rolling of a dice or the prediction of a layman.

However, this is exactly where we see a promising blind spot for younger researchers in our community. If young 
researchers want to try something new, they should build first-class expertise in modelling while maintaining a strong connection to technology. Hal Varian, for instance, has built his career on working at the intersection of information technology and economics (e.g., the seminal work, Shapiro and Varian 1998). Erik Brynjolfsson is another outstanding example of a scientist who currently explains how the digital revolution is transforming our society and economy. Based on scientific arguments, he tries to explain why in the US the median income is no longer rising or why the share of the working population is falling so rapidly (Brynjolfsson and McAfee 2011).

As these two scientists show, there are many important questions at the interface of information technology and other disciplines, and rigorous answers to these important questions have a high publication potential (e.g., Krämer et al. 2018). Accordingly, we argue that we need more experts in this field of research.

In general, there are a number of policy-related topics that need more informed support from the scientific community. For example, there is hardly any research on the General Data Protection Regulation and its economic impact at the firm or country level. However, this information is essential for sound policy making.

Although such blind spots may also be of interest for researchers from the economics or sociology disciplines, these often lack the detailed technological insights to address them. Hence, we believe that the Business and Information Systems Engineering community is much more prone and suited to answering this kind of questions.

Another blind spot is the development of new methods to deal with this new kind of questions. Very often we lack the right instruments to tackle important questions, so that the development of new or the improvement of existing instruments is of imperative necessity. These instruments need to ensure the fundamental scientific principles (i.e., verifiability, reproducibility, and generalizability) that are paramount in all scientific disciplines (Bichler et al. 2014). If we manage to develop new and innovative instruments and methods to address questions at the intersection of information technology and economy or society, our contribution to academia will go beyond our discipline and serve also neighboring scientific communities.

For example, we certainly all agree that distributed ledger technologies could challenge the prevalent intermediation business model in at least some industries. The conceptual story of the technology is straightforward and can be explained in one minute. What we lack, however, are instruments to better understand such an important phenomenon and to make informed predictions how this technology could alter companies or even entire industries. Instead, our community produces a plethora of papers about application scenarios for blockchains, although it is already crystal clear that this technology can be valuable whenever a distributed database is of particular use.

Similarly, we also see many prediction papers applying different Machine Learning (ML) approaches, although we currently do not fully understand the cases in which novel ML methods are superior to classical approaches using regression or simple decision trees. Many of these papers often focus on parameter-tweaking of algorithms to make excellent black-box classifications and predictions. However, the results rarely provide generalizable insights. Accordingly, we argue that it is essential to gain a sound understanding of how machine learning can be of real use for business practice. In this context, the interaction between artificial intelligence and human decision makers seems to be a fruitful playing field for researchers in our community.

Interestingly, accurate classifications are often not enough for decision makers. A good understanding of the context is essential to distinguish between correlation and causality. As thought leaders like Turing Award winner Judea Pearl (e.g., Pearl and Mackenzie 2018) note, decision makers urge to understand the mechanisms behind classifications and are also looking for reliable causal effects. A significant relation between $\mathrm{X}$ and $\mathrm{Y}$ might be good enough for meaningful predictions. However, decision makers need to be sure that managing $X$ will have a causal impact on $\mathrm{Y}$. If $\mathrm{X}$ is only correlated to $\mathrm{Y}$ without having a causal effect, $\mathrm{X}$ cannot be an effective management factor, and the results from machine learning can be misleading. For example, eating more ice-creams will not increase the temperature and carrying umbrellas will not help to circumvent draught. To address these shortcomings, it might be beneficial to complement ML with additional laboratory experiments and domain knowledge.

In the context of AI and ML, it would also be interesting to understand the societal and individual impacts of such large-scale classification procedures. In fact, we are talking about engineered prejudices which are appraised as - at least in the case of humans ethically undesirable. Thus, algorithmic unfairness will be another topic that needs to be addressed in the near future. The topic of Responsible Data Science (van der Aalst et al. 2017) focusing on Fairness, Accuracy, Confidentiality, and Transparency (FACT) is a prime example of a blind spot that requires more attention from our community.

Finally, we also need to integrate the atomic insights generated by individual academic papers to create business process value chains. In marketing, for example, there are many insights into when and how to optimally approach new customers, or conduct successful add-on selling. However, all these papers focus on very specific individual elements of the customer relationship. What businesses need is a storyboard that helps them serve different 
customers segments or even individual customers throughout their customer lifecycle. Such a storyboard should, of course, incorporate all latest insights for the specific touch points throughout the journey. Only a holistic process chain that integrates all these independent insights would be of highest value for practitioners.

In a broad community such as BISE, everybody will see different blind spots. More so since the list of blind spots we have mentioned is certainly not exhaustive. It is always interesting to see how research areas are growing and shrinking, and how they are distributed across the globe. Interestingly, some topics are growing very fast although it remains unclear what the sustained need is. In contrast, other topics are dwindling and then become reinvented, while funding also influences scientific trends in unclear ways (e.g., NSF funding in the US and EU Horizon 2020 in the EU).

A proverb which is widely attributed to Heraclitus - and which has also been emphasized by our new EiC in his last editorial - is "The only constant in life is change". Because this fact certainly holds for our field, we as a community need to re-invent ourselves from time to time.
Therefore, the education and creativity of young academics in our field are of particular importance and we - as more senior colleagues - need to support them, especially if they are eager to go off the beaten tracks.

\section{References}

Bichler M, Heinzl A, Winter R (2014) Diversity and quality in BISE research. Bus Inf Syst Eng 6(6):313-316

Brynjolfsson E, McAfee A (2011) Race against the machine: how the digital revolution is accelerating innovation, driving productivity, and irreversibly transforming employment and the economy. Digital Frontier Press, Lexington

Krämer J, Schnurr D, Wohlfarth M (2018) Winners, losers, and Facebook: the role of social logins in the online advertising ecosystem. Manag Sci. https://doi.org/10.1287/mnsc.2017.3012

Pearl J, Mackenzie D (2018) The book of why: the new science of cause and effect. Basic Books, New York

Shapiro C, Carl S, Varian HR (1998) Information rules: a strategic guide to the network economy. Harvard Business Press, Cambridge

van der Aalst W, Bichler M, Heinzl A (2017) Responsible data science. Bus Inf Syst Eng 59(5):311-313 